\title{
GUIDELINE
}

\section{Consensus statement: Management of} $\checkmark$ drug-induced liver injury in HIV-positive patients treated for TB

E Jong, ${ }^{1}$ MD, PhD; F Conradie, ${ }^{1}$ MB BCh, DTM\&H, Dip HIV Man; R Berhanu, ${ }^{2}$ MD, DTM\&H, Dip HIV Man; A Black, ${ }^{3}$ BSc, MB BCh, FCP (SA), Cert Pulm (SA); M-A John, ${ }^{4} \mathrm{MB}$ ChB, FCPath (Micro), Dip HIV Man, DTM\&H; G Meintjes, ${ }^{5}$ MB ChB, MRCP (UK), FCP (SA), Dip HIV Man, MPH, PhD; C Menezes, ${ }^{6}$ MD, MMed, Dip HIV Man, DTM\&H, FCP (SA)

\author{
${ }^{1}$ Clinical HIV Research Unit, Department of Medicine, Faculty of Health Sciences, University of the Witwatersrand, \\ Johannesburg, South Africa \\ ${ }^{2}$ TB Focal Point, Right to Care, Johannesburg, South Africa \\ ${ }^{3}$ Wits Reproductive Health and HIV Institute, Department of Medicine, Faculty of Health Sciences, University of the Witwatersrand, \\ Johannesburg, South Africa \\ ${ }^{4}$ Durban International Clinical Trials Unit, University of KwaZulu-Natal, Durban, South Africa \\ ${ }^{5}$ Institute of Infectious Disease and Molecular Medicine, and Department of Medicine, Faculty of Health Sciences, \\ University of Cape Town, South Africa \\ ${ }^{6}$ Division of Infectious Diseases, Department of Medicine, Faculty of Health Sciences, University of the Witwatersrand, \\ Johannesburg, South Africa
}

Corresponding author: F Conradie (fconradie@witshealth.co.za)

Disclaimer. This consensus statement represents a consultative process with the authors and other experts in the field. At the time of publishing, this represented the best possible advice based on the data and experience of the group. We acknowledge that as more information is collected, both from randomised controlled trials and cohort data, this consensus statement will need to be updated. There will be at least a biennial review of the document.

Drug-induced liver injury (DILI) in HIV/tuberculosis (TB) co-infected patients is a common problem in the South African setting, and re-introduction of anti-TB drugs can be challenging for the healthcare worker. Although international guidelines on the re-introduction of TB treatment are available, the definition of DILI is not uniform, management of antiretroviral therapy (ART) in HIV co-infection is not mentioned, and the guidance on management is not uniform and lacks a practical approach. In this consensus statement, we summarise important aspects of DILI and provide practical guidance for healthcare workers for different patient groups and healthcare settings on the re-introduction of anti-TB drugs and ART in HIV/TB co-infected individuals presenting with DILI.

S Afr J HIV Med 2013;14(3):113-119. DOI:10.7196/SAJHIVMED.976

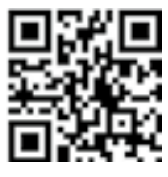

\section{Background}

Globally, 34 million (31.4 - 35.9 million) people were living with $\mathrm{HIV}$ at the end of 2011. South Africa (SA) carries the highest burden, with 5.6 million people infected. ${ }^{[1]}$ A major scale-up of public sector antiretroviral therapy (ART) has seen 1.6 million people start such therapy, and an increasing proportion of eligible individuals initiate treatment. ${ }^{[2]}$ SA has the third highest tuberculosis (TB) burden in the world and the fourth highest multidrug-resistant (MDR) TB rate. ${ }^{[3]}$ An estimated $60-80 \%$ of new TB cases in SA are HIV co-infected. ${ }^{[3,4]}$ $\mathrm{TB}$ remains an important cause of morbidity and mortality among HIV-infected individuals, and undiagnosed TB is a major cause of death in the first months of ART. ${ }^{[5]}$ Adherence to TB treatment is an important determinant of a favourable treatment outcome, and those who default treatment, especially if soon after treatment initiation, continue to be a source of infection and transmission within the community. ${ }^{[6,7]} \mathrm{A}$ twofold higher relative risk of adverse events has been reported in HIV-infected individuals. ${ }^{[8,9]}$

Drug-induced liver injury (DILI) is a well-recognised adverse drug reaction of TB treatment and ART. DILI complicates TB treatment in $5-33 \%$ of patients. First-line anti-TB drugs associated with hepatotoxicity are isoniazid (INH), rifampicin (RIF) and pyrazinamide (PZA). Depending on the regimen, DILI develops in $9-30 \%$ of patients receiving ART. ${ }^{[11]}$ Transaminitis can be associated with most ART, although nevirapine (NVP) is associated with the highest risk of DILI. A retrospective, observational study from SA showed an in-hospital and 3-month mortality among TB treatment or ART-associated DILI patients of $27 \%$ and $35 \%$, respectively. ${ }^{[12]}$

Given the high HIV prevalence and TB incidence, DILI in TB/HIV co-infected patients is a common problem in the SA setting, and the re-introduction of anti-TB drugs can 
be challenging for the healthcare worker. Although international guidelines on the re-introduction of $\mathrm{TB}$ treatment are available, the definition of DILI is not uniform, management of ART in HIV co-infection is not mentioned, and the guidance on management is not uniform and lacks a practical approach. In this consensus statement, we aim to summarise some important aspects of DILI and provide practical guidance for healthcare workers, for different patient groups and healthcare settings, on the re-introduction of anti-TB drugs and ART in HIV/TB co-infected individuals presenting with DILI.

\section{Mechanism of DILI}

Hepatotoxicity may result from direct toxicity of the primary compound, a metabolite, or from an immunologically mediated response, affecting hepatocytes, biliary epithelial cells and/or liver vasculature. Predictable DILI is generally characterised by certain doserelated injury and tends to occur rapidly. Injurious free radicals cause hepatocyte necrosis in zones furthest away from the hepatic arterioles. Unpredictable or idiosyncratic reactions account for most types of DILI. These hypersensitivity reactions occur mostly independent of dose and relatively rarely for each drug, and may result in hepatocellular injury and/or portal tract inflammation with cholestasis.

RIF may occasionally cause dose-dependent interference with bilirubin uptake, resulting in subclinical, unconjugated hyperbilirubinaemia or jaundice without hepatocellular damage. ${ }^{[10]}$ This may be transient and occur early in treatment or in some individuals with preexisting liver disease. ${ }^{[10]}$ Hepatocellular injury appears to be rare. PZA may induce both dose-dependent and idiosyncratic hepatotoxicity. PZA may induce hypersensitivity reactions with eosinophilia and liver injury or granulomatous hepatitis in a limited number of cases. INH is cleared mostly by the liver, primarily by acetylation by $\mathrm{N}$-acetyltransferase 2. Mono-acetyl hydrazine is one of the main metabolites; its reactive metabolites are probably toxic to liver tissue through free radical generation. INH hepatotoxicity occurs generally within weeks to months, rather than days of starting the drug, as seen with hypersensitivity reactions. Unlike a classic hypersensitivity reaction, INH rechallenge does not always elicit a rapid recurrence of hepatotoxicity. ${ }^{[10]}$

Hepatotoxicity due to ART may be a result of immune restoration and the development of acute liver injury related to an enhanced immune response to $\mathrm{TB}$ or hepatitis $\mathrm{B}$ virus (HBV) immune reconstitution inflammatory syndrome (IRIS). HBV-IRIS presents as an acute hepatocellular injury, whereas TB-IRIS presents with an obstructive picture. Nucleoside reverse transcriptase inhibitors (NRTIs) are associated with a risk of mitochondrial toxicity and hepatic steatosis. Non-nucleoside reverse transcriptase inhibitors (NNRTIs) are also associated with hepatotoxicity. The hypersensitivity reaction observed with NVP can also include severe transaminitis. ${ }^{[1]}$ NVP-DILI may be accompanied by a rash and fever. Hepatotoxicity is also observed with efavirenz (EFV), but the mechanism seems to be different. ${ }^{[13]}$ The mechanism of protease inhibitor (PI)-associated hepatotoxicity is not known. Some studies have suggested an impaired drug metabolism, especially in individuals with underlying liver disease. ${ }^{[14]}$

To complicate matters, the HIV/TB co-infected patient is often exposed to other potentially hepatotoxic drugs and is prone to conditions associated with liver injury. Co-trimoxazole may be prescribed as preventive therapy or treatment for Pneumocystis jirovecii or toxoplasmosis. Cholestatic jaundice and hepatic necrosis are known side-effects and could be part of a systemic drug hypersensitivity syndrome that occurs independent of plasma drug concentrations. ${ }^{[15]}$ Bacterial sepsis can also induce liver injury by various mechanisms, including the release of peroxynitrite by leucocytes, resulting in damage to the cell membranes; apoptosis of lymphocytes and gastrointestinal epithelial cells; and tissue ischaemia due to microvascular obstruction triggered by the release of pro-inflammatory mediators. ${ }^{[16]}$ The pattern is generally mixed with increased bilirubin levels. TB itself and TB-IRIS can involve the liver. Hepatic TB-IRIS is probably more common than is appreciated clinically. Approximately $56 \%$ of patients with TB-IRIS have clinical hepatomegaly. ${ }^{[17]}$ The hepatomegaly in TB-IRIS is frequently tender and patients may have symptoms of right-upper quadrant pain, nausea and vomiting. ${ }^{[18]}$ The typical pattern of liver enzyme abnormality is mixed with moderate elevation of transaminases, but a far more significant rise in the canalicular enzymes. ${ }^{[18]}$ Gammaglutamyl transferase (GGT) is substantially more elevated in TB-IRIS patients than in HIV-negative patients with $\mathrm{TB}$ involving the liver. While bilirubin may increase, clinical jaundice is uncommon. ${ }^{[18]}$

Differentiating hepatic TB-IRIS from DILI attributed to a TB drug, ART or co-trimoxazole, poses a clinical conundrum. Unfortunately, there are no features absolutely predictive of either IRIS or DILI. Tender hepatomegaly, the preponderance of an elevation of the canalicular enzymes, the absence of jaundice, maintained synthetic liver function and TB-IRIS features in other organ systems may suggest TB-IRIS rather than DILI. The more definitive diagnostic route is to perform liver biopsy. However, this procedure is invasive and not readily available. Histological features observed in patients with TB-IRIS include significantly increased numbers of granulomas per liver core with abundant eosinophils palisading around these granulomas. ${ }^{[18]}$ When there is doubt as to whether the liver function test (LFT) abnormalities represent TB-IRIS or DILI, it is safer to manage as DILI and monitor the response.

\section{Risk factors for DILI}

Risk factors for DILI in patients receiving TB treatment or ART are well described in the literature. The most important risk factors in individuals receiving TB treatment are: age $>35$ years; being a child; perhaps female gender; hepatitis B surface antigen ( $\mathrm{HBsAg}$ ) positivity, particularly if eAg-positive; use of alcohol; slow acetylator status; extensive TB disease; increase in baseline alanine transaminase (ALT); malnutrition; hepatitis $\mathrm{C}$ virus (HCV) co-infection; and HIV co-infection. ${ }^{\left[{ }^{[0,19,20]}\right.}$ However, most data on TB-DILI in HIV-infected individuals come from observational studies or treatment trials, generally before the advent of effective ART. Definitions of hepatotoxicity and methodology varied among studies, and most lacked HIV-negative control groups. The overall influence of HIV infection alone on DILI during TB treatment was difficult to assess, but appeared to be slight, with the exception of one study where other confounding causes of DILI were present, such as injection drug use, alcoholism, ART and viral hepatitis. ${ }^{[10]}$ One study from Ethiopia looked at TB-DILI in a population grouped by HIV status; ${ }^{[2]}$ i.e. biochemical TBDILI in HIV-positive and HIV-negative individuals. HIV-infection (odds ratio (OR) 3.6; 95\% confidence interval (CI) 1.5 - 8.5), concomitant drug

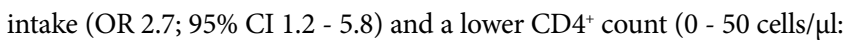
OR 21.3 (95\% CI 2.2 - 204.9); CD4+ 51 - 100 cells/ $\mu$ : OR 5.2 (95\% CI 0.6 46.0); $\mathrm{CD}^{+} 101$ - 200 cells/ $\mu \mathrm{l}$ : OR 4.3 (95\% CI 0.4 - 42.4)) relative to a CD $4^{+}$ count $>200$ cells/ $\mu \mathrm{l}$, were identified as factors significantly associated with biochemical TB-DILI. ${ }^{[21]}$ Risk factors associated with severe ART-related 
Table 1. Overview of monitoring advice and cut-off levels for DILI according to existing guidelines

\begin{tabular}{|c|c|c|}
\hline Authority & $\begin{array}{l}\text { Monitoring in the presence of } \\
\text { risk factors (especially liver } \\
\text { diseases) }\end{array}$ & Cut-off levels for DILI and stopping drugs \\
\hline $\operatorname{ATS}^{[10]}$ & Yes & ALT > $200 \mathrm{IU} / 1$, or ALT >120 IU/1 with symptoms \\
\hline $\mathrm{BTS}^{[29]}$ & Yes & ALT or AST $>200 \mathrm{IU} / \mathrm{l}$, rise in bilirubin \\
\hline ERS, WHO, IUATLD ${ }^{[30]}$ & - & ALT or AST $>200 \mathrm{IU} / 1$, icteric patient \\
\hline $\mathrm{HKTBS}^{[31]}$ & Yes & ALT $>200 \mathrm{IU} / \mathrm{l}$, bilirubin $>40 \mu \mathrm{mol} / \mathrm{l}$ \\
\hline
\end{tabular}

\section{Table 2. DILI definition advocated in the SA setting}

- ALT level >120 IU/l and symptomatic (nausea, vomiting, abdominal pain, jaundice); or

- ALT level $>200 \mathrm{IU} / \mathrm{l}$ and asymptomatic; or

- Total serum bilirubin concentration $>40 \mu \mathrm{mol} / \mathrm{l}$

hepatotoxicity were a baseline elevation in serum aminotransferases, concomitant hepatotoxic medication, thrombocytopenia, renal insufficiency, chronic HBV or HVC co-infection and female gender. ${ }^{[2-25]}$

\section{Definition of DILI}

The definition of TB-DILI, according to the commonly used management guidelines, is summarised in Table 1. Asymptomatic transaminase elevations occur in $20 \%$ of patients started on standard anti-TB regimens prior to, or immediately after the start of treatment. Usually these elevations resolve spontaneously. ${ }^{[2-28]}$ RIF may occasionally cause dose-dependent interference with bilirubin uptake, resulting in subclinical, unconjugated hyperbilirubinaemia or jaundice without hepatocellular damage. This may be transient and occur early in treatment or in some individuals with pre-existing liver disease.

Although similarities exist between the major guidelines, there are also differences. To achieve a uniform approach to DILI, a clear definition is required. None of the guidelines recommend routine monitoring of liver function in patients in whom there is no reason to suspect liver dysfunction. The definition for DILI in Table 2 has been advocated in the SA setting.

This definition applies to all TB patients including those with HIV co-infection. Although most guidelines suggest baseline LFT and laboratory monitoring of risk groups including HIV-positive individuals, this is generally not performed in low-resource countries with a high TB burden because of additional costs and low detection rates. Nevertheless, a healthcare worker should always enquire whether baseline laboratory parameters are available. In patients with a baseline ALT $>120 \mathrm{IU} / 1$ or total bilirubin $>40 \mu \mathrm{mol} / \mathrm{l}$, it is recommended to start standard TB treatment and monitor closely, or to omit PZA initially in severe cases. If LFTs worsen on TB treatment, an alternative regimen should be started.

Elevated GGT and alkaline phosphatase (ALP) levels are not included in the DILI-definition of any of the major TB guidelines. Cholestasis is frequently observed in HIV-infected individuals and the cause can be multifactorial e.g. TB of the liver, TB-IRIS, drugs, opportunistic infections and fatty liver, but also HIV-related malignancies and biliary tract disease. ${ }^{[32]}$ GGT and ALP levels should not be monitored routinely. Elevations of GGT alone do not reflect liver injury.

\section{Management of TB-DILI: Existing guidelines and studies}

Guidelines on the management of TB and adverse drug reactions have been published by the American Thoracic Society (ATS); the British Thoracic Society (BTS); the Task Force of the European Respiratory Society (ERS), the WHO and the International Union Against Tuberculosis and Lung Disease (IUATLD); and the Hong Kong Tuberculosis Service (HKTBS). ${ }^{[10,29-31,33]}$ Most guidelines are based on expert opinion from a multidisciplinary team supported by literature searches. Good quality studies with adequate sample sizes on the preferred TB treatment regimen in patients presenting with DILI are scarce and will be discussed later. In the case of confirmed moderate or severe drug-induced hepatotoxicity, treatment should be interrupted and re-introduced after hepatotoxicity has resolved. When and how TB drugs should be re-introduced according to the various guidelines is summarised in Table 3 .

However, in the SA setting, HIV/TB co-infected patients with DILI often have significant immune suppression and large mycobacterial loads, and the clinician may initiate a $\mathrm{TB}$ treatment backbone that excludes the first-line hepatotoxic drugs while the LFTs settle and until the patient is ready for rechallenge. Only the BTS guideline ${ }^{[2]}$ provides guidance for these cases. Also, it is not clear what approach should be followed when the healthcare worker fails to re-introduce some of the first-line drugs, especially INH or RIF. The BTS guideline is reasonable and advises consultation with a fully trained physician, which is not always feasible in the Southern African setting.

Few published studies have focused on different re-introduction regimens of TB drugs. ${ }^{[34-37]}$ All excluded HIV-positive patients and only two were randomised trials. A trial by Tahaoglu et al.$^{[35]}$ randomised 45 patients to INH, RIF, ethambutol (EMB) and streptomycin (STR) by gradually increasing the number and dosage of the drugs $(n=20)$ or the standard regimen (INH, RIF, EMB and PZA) in the same dosage from the start of the rechallenge $(n=25)$. Hepatotoxicity recurred in 0 and $6(24 \%)$ patients $(p=0.021)$, respectively. The time from withdrawal of TB drugs to resolution of hepatotoxicity was 18.7 days (standard deviation \pm 11.4$)^{[35]}$ Sharma et al. ${ }^{[36]}$ randomised 175 patients with 
Table 3. Overview of management of TB-DILI according to existing guidelines

\begin{tabular}{|c|c|c|c|c|c|}
\hline Authority & $\begin{array}{l}\text { Stopping TB } \\
\text { drugs if clinical or } \\
\text { symptomatic hepatitis }\end{array}$ & $\begin{array}{l}\text { When to } \\
\text { restart TB } \\
\text { drugs }\end{array}$ & What TB drugs to start & $\begin{array}{l}\text { Recommended } \\
\text { LFT monitoring on } \\
\text { rechallenge }\end{array}$ & If DILI recurs \\
\hline $\operatorname{ATS}^{[10]}$ & Yes & ALT $<80 \mathrm{IU} / 1$ & $\begin{array}{l}\text { - RIF +/- EMB full dose } \\
\text { - After } 3-7 \text { days INH } \\
\text { (full dose) } \\
\text { - PZA only if mild DILI }\end{array}$ & $\begin{array}{l}\text { Check ALT } 3 \text { - } 7 \\
\text { days after INH } \\
\text { rechallenge }\end{array}$ & $\begin{array}{l}\text { - Stop last drug } \\
\text { added }\end{array}$ \\
\hline $\mathrm{BTS}^{[29]}$ & Yes & $\begin{array}{l}\text { ALT within } \\
\text { normal limits }\end{array}$ & $\begin{array}{l}\text { - STR + EMB (if unwell } \\
\text { or sputum is smear- } \\
\text { positive within } 2 \\
\text { weeks of commencing } \\
\text { treatment) } \\
\text { - INH (dose titration, } \\
\text { every } 2 \text { - } 3 \text { days) } \\
\text { - RIF (dose titration, } \\
\text { every } 2 \text { - } 3 \text { days) } \\
\text { - PZA (dose titration, } \\
\text { every } 2 \text { - } 3 \text { days) }\end{array}$ & $\begin{array}{l}\text { - Daily } \\
\text { monitoring } \\
\text { of LFT }\end{array}$ & $\begin{array}{l}\text { - Stop offending } \\
\text { drug, alternative } \\
\text { regimen advised } \\
\text { by fully trained } \\
\text { physician }\end{array}$ \\
\hline $\begin{array}{l}\text { ERS, WHO, } \\
\text { IUATLD }^{[30]}\end{array}$ & Yes & $\begin{array}{l}\text { LFT within } \\
\text { normal limits }\end{array}$ & $\begin{array}{l}\text { - Start all drugs at full } \\
\text { dosage }\end{array}$ & $\begin{array}{l}\text { - } \text { LFT } \\
\text { monitoring (no } \\
\text { recommendation } \\
\text { on frequency) }\end{array}$ & $\begin{array}{l}\text { - Stop all drugs, } \\
\text { start STR + EMB } \\
\text { and start other } \\
\text { drugs one at a } \\
\text { time }\end{array}$ \\
\hline $\mathrm{HKTBS}^{[31]}$ & Yes & - & - & - & - \\
\hline
\end{tabular}

\section{Table 4. TB treatment regimen for patients with drug-susceptible TB when a first-line drug is omitted}

\begin{tabular}{lll}
\hline Drug omitted & Intensive phase & Continuation phase \\
\hline RIF & INH, MOX, EMB, STR $\times 2$ months ${ }^{*}$ & INH, MOX, EMB $\times 16$ months \\
INH & RIF, MOX, EMB $\times 2$ months ${ }^{*}$ & RIF, MOX, EMB $\times 10$ months \\
PZA & RIF, INH, EMB $\times 9$ months \\
& \\
& \\
& TB = tuberculosis; RIF = rifampicin; INH = isoniazid; MOX = moxifloxacin; EMB = ethambutol; STR = streptomycin; PZA = pyrazinamide.
\end{tabular}

TB-DILI to one of three predefined re-introduction regimens. In the first arm, patients were given INH, RIF and PZA simultaneously at full dosage from day 1 . In the second arm, drugs were re-introduced according to the ATS guideline with RIF at maximum dosage from day $1, \mathrm{INH}$ at maximum dosage from day 8 , and PZA at maximum dosage from day 15 . In the third arm, drugs were administered in accordance with the BTS guidelines with $100 \mathrm{mg}$ /day INH from day 1, (maximum dosage from day 4), $150 \mathrm{mg}$ /day RIF from day 8 (maximum dosage from day 11) and $500 \mathrm{mg} /$ day PZA from day 15 (maximum dosage from day 18). The recurrence rates were not significantly different between the three arms (around 10\%). The median time from withdrawal of the TB drugs to resolution of hepatotoxicity was 18 days (interquartile range $14-28) \cdot{ }^{[36]}$ Some studies have looked at the use and safety of fluoroquinolones in TB-DILI patients. In these studies, the use of fluoroquinolones did not result in additional hepatotoxicity; however, the potential hepatotoxicity associated with fluoroquinolones (although infrequent) is something of which clinicians should be aware. ${ }^{[38,39]}$ Szklo et al. ${ }^{[38]}$ reported a cure rate of $85 \%$ and no treatment failures or relapses after 2 years of follow-up with fluoroquinolone regimens in patients who had DILI.

\section{Management of TB-DILI in a high HIV burden setting: Recommendations}

- Check TB diagnosis.

- Check results of TB culture and drug susceptibility testing.

- Reconsider TB diagnosis if not confirmed. If the patient has had a clinical response to the $\mathrm{TB}$ treatment including weight gain, improvement of symptoms that were compatible with a TB diagnosis and/or chest X-ray improvements, then the diagnosis can be assumed to be correct.

- Check if the patient is on the intensive or continuation phase of TB treatment.

- Consider other causes such as chronic alcohol consumption, preexisting liver disease, viral hepatitis, other hepatotoxic medications, TB involvement of the liver, IRIS and bacterial sepsis. 


\subsection{Group 1: Intensive phase of TB treatment}

\subsubsection{Mild DILI: Clinically well with elevated ALT $<\mathbf{2 0 0} \mathrm{IU} / \mathrm{l}$ and total bilirubin $<\mathbf{4 0} \boldsymbol{\mu m o l} / \mathrm{I}$}

- Continue TB drugs in the event of confirmed or probable TB.

- Continue ART if the patient is receiving ART.

- Repeat ALT and bilirubin in one week.

- If ALT and bilirubin have improved or normalised, stop laboratory monitoring.

- If ALT and bilirubin remain elevated but stable for 4 consecutive weeks, consider the other causes listed above, abdominal sonar and referral for further workup of liver dysfunction.

- If ALT and bilirubin increase further and meet the DILI definition, then move on to the relevant section below.

\subsubsection{Moderate DILI: Clinically well and elevated ALT $>200$ IU/I irrespective of total bilirubin}

- Discontinue the standard TB regimen.

- Start STR, moxifloxacin (MOX) and EMB (note: STR is contraindicated if the glomerular filtration rate (GFR) is $<60 \mathrm{ml} / \mathrm{min}$ ).

- Discontinue co-trimoxazole prophylaxis and other hepatotoxic drugs.

- Stop ART. If the patient is on an NNRTI-based regimen, stop the NNRTI first and the NRTIs after $5-7$ days. If the patient is on a PI-based regimen, stop all drugs at once. If the patient has been on a stable ART regimen for $>6$ months, consider continuing the therapy, as it is less likely that ART is the cause.

- Repeat ALT and bilirubin in 2 - 3 (inpatient) or 7 days (outpatient).

- When ALT is $<100 \mathrm{IU} / 1$ and total bilirubin is normal, start the rechallenge.

- Day 1: RIF 450 or 600 mg daily, depending on weight.

- Day 3: Check ALT.

- Day 4 - 6: Add 300 mg INH daily.

- Day 7: Check ALT.

- Day 8: Consider a PZA rechallenge (especially in the case of TB meningitis or intolerance/resistance to other drugs) and check ALT at day 10

- Once rechallenged, follow the guidance in Table 4.

- Monitor ALT weekly for 4 weeks after the rechallenge.

\subsubsection{Moderate DILI: Isolated jaundice (ALT <120 IU/I and total bilirubin $>\mathbf{4 0} \mathbf{\mu m o l} / \mathrm{l}$ )}

- Continue ART.

- Stop co-trimoxazole prophylaxis if ALP and GGT are also elevated.

- Stop RIF (RIF is the most likely cause of isolated jaundice).

- Continue INH, PZA and EMB and add MOX.

- Repeat ALT and bilirubin after 7 days. If bilirubin does not settle, then consult an expert.

- If the bilirubin settles, then follow the guidance in Table 4.

- The clinician may consider a full-dose RIF rechallenge 2 - 3 weeks after stopping RIF.

- Repeat ALT and bilirubin after 7 days. If ALT and bilirubin are stable, then stop MOX and continue with standard RIF, INH, PZA and EMB for 2 months, followed by 4 months of RIF and INH.

- If bilirubin increases to $>40 \mu \mathrm{mol} / \mathrm{l}$, stop RIF and continue INH, PZA, EMB and MOX. Follow the guidance in Table 4.

- Monitor ALT and bilirubin weekly for 4 weeks after the rechallenge.

- If ALT increases to > 120 IU/l, perform abdominal ultrasound and refer for further investigation. Follow the guidance in sections 6.1.1 or 6.1.2.

\subsubsection{Severe DILI: Clinically not well (nausea, vomiting, abdominal pain), meets DILI definition (Table 2)}

- Admit the patient.

- Consult an infectious diseases or TB-treatment specialist, if available.

- Assess liver synthetic function with an international normalised ratio (INR) and monitor blood glucose (hypoglycaemia can complicate liver failure).

- Stop standard TB treatment, co-trimoxazole prophylaxis and all other hepatotoxic drugs.

- If the patient is on an NNRTI-based regimen, stop the NNRTI first and the NRTIs after 5 - 7 days. However, if the patient is in liver failure, stop all ART immediately.

- If the patient is on a PI-based regimen, stop all drugs at once.

- Start EMB, STR and MOX (note: STR is contraindicated if the GFR is $<60 \mathrm{ml} / \mathrm{min}$ ).

- Repeat ALT and bilirubin after 2 - 3 days.

- Rechallenge TB drugs when ALT is $<100 \mathrm{IU} / 1$ and bilirubin is normal.

- Day 1: RIF 450 or $600 \mathrm{mg}$ daily depending on weight.

- Day 3: Check ALT (should improve or become stable).

- Day 4 - 6: Add 300 mg INH daily.

- Day 7: Check ALT (ALT should improve or become stable).

- Stop STR and follow the guidance in Table 4.

- Consider PZA re-challenge (especially in the case of TB meningitis or intolerance/resistance to other drugs).

- Monitor ALT weekly for 4 weeks after rechallenge.

\subsubsection{Management if RIF is not tolerated after rechallenge}

- Stop RIF.

- Check ALT after 2 - 3 days.

- If ALT is $<100 \mathrm{IU} / \mathrm{l}$ and bilirubin is normal, add $300 \mathrm{mg} \mathrm{INH}$ daily.

- Check ALT after 2 - 3 days.

- If ALT is stable, follow the guidance in Table 4.

- If ALT is worsening, stop INH and continue treatment in consultation with an infectious diseases or TB-treatment specialist.

- Consider PZA rechallenge where RIF has not been tolerated.

- Monitor ALT weekly for 4 weeks after rechallenge.

\subsubsection{Management if INH is not tolerated after rechallenge}

- Stop INH.

- Check ALT after 2 - 3 days.

- Continue RIF, MOX and EMB for 12 months in total.

- Consider PZA rechallenge in this setting where INH has not been tolerated.

- Monitor ALT weekly for 4 weeks after rechallenge.

\subsection{Group 2: Continuation phase of TB} treatment

- Confirm the start date and response to ART/TB treatment.

- Consider other diagnoses.

- If asymptomatic with elevated ALT $<200 \mathrm{IU} / \mathrm{l}$ and bilirubin $<40$ $\mu \mathrm{mol} / \mathrm{l}$, then follow the approach in 6.1.1.

- If the patient has isolated jaundice (ALT $<120 \mathrm{IU} / 1$ and total bilirubin $>40 \mu \mathrm{mol} / \mathrm{l}$ ), stop RIF, continue INH, EMB and MOX. Follow the guidance in Table 4. 
- Continue ART. Stop co-trimoxazole prophylaxis if ALP and GGT are also elevated.

- The clinician may consider full-dose RIF rechallenge 2 - 3 weeks after stopping RIF.

- If the patient has moderate DILI (asymptomatic and elevated ALT > 200 IU/l irrespective of total bilirubin) or severe DILI, then discontinue the standard TB regimen, co-trimoxazole prophylaxis and other hepatotoxic drugs.

- If the patient has severe DILI, assess liver synthetic function with an INR and monitor blood glucose.

- Stop ART. If the patient is on an NNRTI-based regimen, stop the NNRTI first and the NRTIs after 5 - 7 days (however, if the patient is in liver failure, stop all ART immediately). If the patient is on a PI-based regimen, stop all drugs at once.

- Monitor ALT and bilirubin in 2 - 3 days (inpatient) or 7 days (outpatient).

- Rechallenge TB drugs if ALT is $<100 \mathrm{IU} / \mathrm{l}$ and bilirubin is normal.

- Day 1: 450 - 600 mg RIF daily depending on weight.

- Day 3: Check ALT (ALT should be stable).

- Day 4 - 6: Add 300 mg INH daily.

- Day 7: Check ALT.

- Continue RIF and INH to complete the continuation phase.

- If ALT and/or bilirubin worsen after RIF rechallenge, treat with INH, EMB and MOX. Further diagnostic tests and treatment duration in consultation with an infectious diseases or TB-treatment specialist may be needed.

- If ALT and/or bilirubin worsen after INH rechallenge, then treat with RIF, EMB and MOX. Further diagnostic tests and treatment duration in consultation with an infectious diseases or TB-treatment specialist may be needed.

- Monitor ALT weekly for 4 weeks after rechallenge.

\subsection{General comments for all TB-DILI patients}

- The re-introduction of first-line TB drugs in patients with TB-DILI is preferred over the use of second-line drugs.

- Rechallenge is not recommended for those who have had fulminant hepatitis (defined as hepatic encephalopathy with coagulopathy). Once ALT is $<100 \mathrm{IU} / \mathrm{ml}$ and bilirubin has normalised, treat with an MDR-TB regimen, avoiding PZA and substituting PZA with EMB (i.e. STR or kanamycin, EMB, MOX, ethionamide and terizidone).

- If the patient cannot tolerate MOX, high-dose levofloxacin should be used. Ciprofloxacin should not be used in the treatment of TB.

- If STR is not available, then kanamycin or amikacin should be used.

- If the intensive phase of TB treatment is interrupted for any period, restart the full treatment course starting from the day that the alternative $\mathrm{TB}$ regimen is successfully re-introduced.

- Re-challenge with PZA was previously not recommended, but a recent trial has shown that most patients tolerate it. ${ }^{[36]} \mathrm{PZA}$ re-challenge should be considered in patients with severe TB (e.g. miliary, meningitis), drug resistance or intolerance to INH or RIF. Transaminase levels, especially ALT, should be monitored frequently (e.g. 3 times weekly) during rechallenge and weekly for a month following rechallenge.

- Patients with low body weight $(<40 \mathrm{~kg})$ should have their maximal doses calculated according to body weight.
- Monitor vision in all patients requiring a modified regimen, including signs of EMB toxicity.

- ART is indicated for all TB/HIV patients independent of $\mathrm{CD} 4^{+}$cell count.

\subsection{Rechallenge or initiation of ART \\ 6.4.1 Patients who develop DILI while receiving TB medication only, not on ART}

\subsubsection{ART-naive}

- $\mathrm{CD}^{+}$count $<100$ cells/ $\mu \mathrm{l}$ : start ART as soon as the complete standard or alternative regimen of TB drugs is re-introduced. ${ }^{[40]}$

- $\mathrm{CD}^{+}$count $>100$ cells/ $\mu \mathrm{l}$ : ART can be delayed until after the intensive phase of TB treatment, unless the patient has other serious HIVrelated conditions (e.g. Kaposi's sarcoma or HIV encephalopathy). ${ }^{[40]}$

- After ART initiation, monitor ALT every 2 weeks for 2 months.

\subsubsection{ART-experienced}

- Collect information on treatment history, treatment response and reason for treatment interruption.

- Discuss the appropriate regimen with an infectious diseases or HIVtreatment specialist.

- After ART initiation, monitor ALT every 2 weeks for 2 months.

\subsubsection{Patients who develop DILI while receiving TB treatment and ART}

- If DILI developed on an NNRTI-based regimen with NVP, then rechallenge with an NNRTI-based regimen with EFV after the TB drug rechallenge. ${ }^{[13,40]}$

- If DILI developed on NNRTI-based regimen with EFV, then rechallenge EFV in the case of mild DILI after the TB drug rechallenge. In the case of severe or recurrent DILI, start a PI-based regimen with lopinavir/ritonavir (LOP/r) (with dose adjustment if receiving RIF).

- If the patient has mild DILI on EFV and LFTs resolve within 5 - 7 days after interrupting EFV, the clinician may consider restarting EFV before the TB drug rechallenge in order to avoid stopping ART.

- If DILI developed on PI-based regimen with double-dose LOP/r, then the preferred approach is to replace RIF with $150 \mathrm{mg}$ rifabutin on alternate days and use this with atazanavir/ritonavir (or a standard dose of LOP/r). If this option is not available and double-dose LOP/r with RIF is used, then this should be recommenced with slow-dose escalation over 2 weeks: i.e. day 1 - 400/100 mg 12-hourly; day 7 600/150 mg 12-hourly; day $14-800 / 200$ mg 12-hourly. This should be done after the TB drug rechallenge. Note: There is no need for doubledose $\mathrm{LOP} / \mathrm{r}$ if there is no RIF in the final TB treatment regimen.

- In the case of all other regimens including newer PIs or drugs from other drug classes, discuss the approach with an infectious diseases or HIV-treatment specialist.

- After ART rechallenge, monitor ALT every 2 weeks for 2 months.

\subsection{Rechallenge of co-trimoxazole}

Co-trimoxazole therapy should not be rechallenged in HIV/TB patients taking primary or secondary co-trimoxazole prophylaxis and who experience severe liver toxicity. Dapsone should be used instead, but is less effective than co-trimoxazole in preventing $P$. jirovecii pneumonia and also lacks the broad antimicrobial activity of co-trimoxazole. It is therefore desirable to attempt rechallenge of co-trimoxazole in 
patients with previous $P$. jirovecii pneumonia and with mild DILI after re-introduction of TB treatment and ART. Monitor ALT and bilirubin weekly for 1 month after rechallenge.

Conflict of interest. All authors submitted conflict of interest disclosure forms. Authors were required to report on financial support received from pharmaceutical companies and medical aids over the previous three years (updated 19 August 2013). F Conradie has received support from Aspen Pharmacare to attend conferences, research support from Janssen Pharacetic, and honoraria for speaking engagements from MSD, and acted as a consultant to Discovery Health. G Meintjes has received honoraria for speaking engagements from Sanofi Aventis and serves as a consultant for Aid for AIDS. R Bernhau, A Black, M-A John and $\mathrm{E}$ Jong report no conflicts of interest.

Acknowledgement. This work is funded by the Southern African HIV Clinicians Society through an educational grant from Atlantic Philanthropies (No. 17178).

\section{References}

1. UNAIDS. Global Report: UNAIDS Report on the Global HIV Epidemic 2012. http:// www.unaids.org/en/media/unaids/contentassets/documents/epidemiology/2012/ gr2012/20121120_UNAIDS_Global_Report_2012_en.pdf (accessed 1 July 2013).

2. National Department of Health. Global AIDS Response Progress Report. Pretoria NDoH, 2012. http://www.info.gov.za/view/DownloadFileAction?id=172746 (accessed 1 July 2013).

3. World Health Organization. WHO Report 2011: Global Tuberculosis Control. Geneva: WHO, 2011.

4. van Halsema CL, Fielding KL, Chihota VN, et al. Trends in drug-resistant tuberculosis in a gold-mining workforce in South Africa, 2002 - 2008. Int J Tuber Lung Dis 2012;16(7):967-793. [http://dx.doi.org/10.5588/ijtld.11.0122]

5. Lawn SD, Harries AD, Meintjes G, et al. Reducing deaths from tuberculosis in antiretroviral treatment programmes in sub-Saharan Africa. AIDS 2012;26(17):21212133. [http://dx.doi.org/10.1097/QAD.0b013e3283565dd1]

6. Cohen MS, Chen YQ, McCauley M, et al. Prevention of HIV-1 infection with early antiretroviral therapy. N Engl J Med 2011;365(6):493-505. [http://dx.doi.org/10.1056/ NEJMoa1105243]

7. Daniel OJ, Oladapo OT, Alausa OK. Default from tuberculosis treatment programme in Sagamu, Nigeria. Niger J Med 2006;15(1):63-67.

8. Breen RA, Miller RF, Gorsuch T, et al. Adverse events and treatment interruption in tuberculosis patients with and without HIV co-infection. Thorax 2006;61(9):791-794 [http://dx.doi.org/10.1136/thx.2006.058867]

9. Marks DJ, Dheda K, Dawson R, Ainslie G, Miller RF. Adverse events to antituberculosis therapy: Influence of HIV and antiretroviral drugs. Int J STD AIDS 2009;20(5):339-345. [http://dx.doi.org/10.1258/ijsa.2008.008361]

10. Saukkonen JJ, Cohn DL, Jasmer RM, et al. An official ATS statement: Hepatotoxicity of antituberculosis therapy. Am J Respir Crit Care Med 2006;174(8):935-952. [http:// dx.doi.org/10.1164/rccm.200510-1666ST]

11. Montessori V, Press N, Harris M, Akagi L, Montaner JS. Adverse effects of antiretroviral therapy for HIV infection. CMAJ 2004;170(2):229-238.

12. Schutz C, Ismail Z, Proxenos CJ, et al. Burden of antituberculosis and antiretroviral drug-induced liver injury at a secondary hospital in South Africa. S Afr Med 2012;102(6):506-511.

13. Mehta U, Maartens G. Is it safe to switch between efavirenz and nevirapine in the event of toxicity? Lancet Infect Dis 2007;7(11):733-738. [http://dx.doi.org/10.1016/ S1473-3099(07)70262-1]

14. Sulkowski MS. Drug-induced liver injury associated with antiretroviral therapy that includes HIV-1 protease inhibitors. Clin Infect Dis 2004;38(suppl 2):S90-S97. [http:// dx.doi.org/10.1086/381444]

15. Hughes WT, LaFon SW, Scott JD, Masur H. Adverse events associated with trimethoprim-sulfamethoxazole and atovaquone during the treatment of AIDSrelated Pneumocystis carinii pneumonia. J Infect Dis 1995;171(5):1295-1301.
16. Shah AA, Patton M, Chishty WH, Hussain A. Analysis of elevated liver enzymes in an acute medical setting: Jaundice may indicate increased survival in elderly patients with bacterial sepsis. Saudi J Gastroenterol 2010;16(4):260-263.

17. Meintjes G, Rangaka MX, Maartens G, et al. Novel relationship between tuberculosis immune reconstitution inflammatory syndrome and antitubercular drug resistance. Clin Infect Dis 2009;48(5):667-676. [http://dx.doi.org/10.1086/596764]

18. Meintjes G, Sonderup M. A practical approach to the diagnosis and management of paradoxical tuberculosis immune reconstitution inflammatory syndrome. Continuing Medical Education 2011;29(10):410.

19. Yew WW, Chau CH, Leung S. Anti-tuberculosis drugs and liver toxicity. Eur Respir J 1996;9(2):389-90.

20. Yew WW, Leung CC. Antituberculosis drugs and hepatotoxicity. Respirology 2006;11(6):699-707.

21. Yimer G, Aderaye G, Amogne W, et al. Anti-tuberculosis therapy-induced hepatotoxicity among Ethiopian HIV-positive and negative patients. PLoS One 2008;3(3):e1809. [http://dx.doi.org/10.1371/journal.pone.0001809]

22. Uberti-Foppa C, De Bona A, Morsica G, et al. Pretreatment of chronic active hepatitis $\mathrm{C}$ in patients coinfected with HIV and hepatitis $\mathrm{C}$ virus reduces the hepatotoxicity associated with subsequent antiretroviral therapy. J Acquir Immune Defic Syndr 2003;33(2):146-152.

23. Martin-Carbonero L, Nunez M, Gonzalez-Lahoz J, Soriano V. Incidence of liver injury after beginning antiretroviral therapy with efavirenz or nevirapine. HIV Clin Trials 2003;4(2):115-120.

24. Servoss JC, Kitch DW, Andersen JW, et al. Predictors of antiretroviral-related hepatotoxicity in the adult AIDS Clinical Trial Group (1989 - 1999). J Acquir Immune Defic Syndr 2006;43(3):320-323. [http://dx.doi.org/10.1097/01. qai.0000243054.58074.59]

25. Wit FW, Weverling GJ, Weel J, et al. Incidence of and risk factors for severe hepatotoxicity associated with antiretroviral combination therapy. J Infect Dis 2002;186(1):23-31. [http://dx.doi.org/100.1086/341084]

26. Girling DJ. The hepatic toxicity of antituberculosis regimens containing isoniazid, rifampicin and pyrazinamide. Tubercle 1978;59(1):13-32.

27. Ormerod LP, Horsfield N. Frequency and type of reactions to antituberculosis drugs: Observations in routine treatment. Tuber Lung Dis 1996;77(1):37-42.

28. Sharifzadeh M, Rasoulinejad M, Valipour F, et al. Evaluation of patient-related factors associated with causality, preventability, predictability and severity of hepatotoxicity during antituberculosis treatment. Pharmacol Res 2005;51(4):353-358. [http://dx.doi. org/10.1016/j.phrs.2004.10.009]

29. Joint Tuberculosis Committee of the British Thoracic Society. Chemotherapy and management of tuberculosis in the United Kingdom: Recommendations 1998. Thorax 1998;53(7):536-548

30. Migliori GB, Raviglione MC, Schaberg $\mathrm{T}$, et al. Tuberculosis management in Europe. Task Force of the European Respiratory Society (ERS), the World Health Organization (WHO) and the International Union against Tuberculosis and Lung Disease (IUATLD) Europe Region. Eur Respir J 1999;14(4):978-992.

31. Tam WW, Leung C, Chan YC. Monitoring for hepatotoxicity during antituberculosis treatment: General recommendations. A Consensus Statement of the Tuberculosis Control Coordinating Committee of the Hong Kong Department of Health and the Tuberculosis Subcommittee of the Coordinating Committee in Internal Medicine of the Hospital Authority, Hong Kong. http://wwwinfogovhk/tb_chest/doc/ Drugheppdf (accessed 1 July 2013).

32. Te HS. Cholestasis in HIV-infected patients. Clin Liver Dis 2004;8(1):213-228.

33. National Department of Health. National Tuberculosis Management Guidelines. Pretoria: DoH, 2011.

34. Singh J, Garg PK, Tandon RK. Hepatotoxicity due to antituberculosis therapy. Clinical profile and reintroduction of therapy. J Clin Gastroenterol 1996;22(3):211-214.

35. Tahaoglu K, Atac G, Sevim T, et al. The management of anti-tuberculosis druginduced hepatotoxicity. Int J Tuberc Lung Dis 2001;5(1):65-69.

36. Sharma SK, Singla R, Sarda P, et al. Safety of 3 different reintroduction regimens of antituberculosis drugs after development of antituberculosis treatment-induced hepatotoxicity. Clin Infect Dis 2010;50(6):833-839. [http://dx.doi.org/10.1086/650576]

37. Agal S, Baijal R, Pramanik S, et al. Monitoring and management of antituberculosis drug induced hepatotoxicity. J Gastroenterol Hepatol 2005;20(11):1745-1752. [http:// dx.doi.org/10.1111/j.1440-1746.2005.04048.x]

38. Szklo A, Mello FC, Guerra RL, et al. Alternative anti-tuberculosis regimen including ofloxacin for the treatment of patients with hepatic injury. Int J Tuberc Lung Dis 2007;11(7):775-780.

39. Ho CC, Chen YC, Hu FC, et al. Safety of fluoroquinolone use in patients with hepatotoxicity induced by anti-tuberculosis regimens. Clin Infect Dis 2004;48(11):1526-1533. [http://dx.doi.org/10.1086/598929]

40. Meintjes G, Maartens G, Boulle A, et al. Guidelines for antiretroviral therapy in adults by the Southern African HIV Clinicians Society. Southern African Journal of HIV Medicine 2012;13(3):114-133. [http://dx.doi.org/10.7196/SAJHIVMED.862] 